\title{
A cross-sectional study of 2550 amateur cyclists shows lack of knowledge regarding relevant sports nutrition guidelines
}

\author{
I M Sparks1, MBChB, D C Janse van Rensburg1, MD, L \\ Fletcher ${ }^{2}$, PhD, A Jansen van Rensburg ${ }^{1}$, MSc
}

${ }^{1}$ Institute for Sport Exercise Medicine and Lifestyle Research \& Section Sports Medicine, Faculty of Health Sciences, University of Pretoria, Pretoria, South Africa

${ }^{2}$ Department of Statistics, Faculty of Natural and Agricultural Sciences, University of Pretoria, South Africa

Corresponding author: D C Janse van Rensburg (christa.jansevanrensburg@up.ac.za)

Background: Amateur cyclists use a wide variety of supplements and nutritional substances to increase performance in addition to their training.

Objectives: The intended nutritional supplement use, carbohydrate $(\mathrm{CHO})$ use and hydration practices of amateur cyclists before, during and after endurance cycling were analysed. Evidence of ignorance regarding the use of sports supplements and $\mathrm{CHO}$, as well as the disregard of hydration strategy was hypothesised.

Methods: Amateur cyclists, of all age and sex groups, were requested to complete an online survey anonymously on the 2013 Momentum 94.7 Cycle Challenge website, a few days before the event.

Results: Responses were received from 2550 out of 30640 race entrants (8\%); representing a distribution of $75 \%$ males, $25 \%$ females, with the majority between 25 to 45 years old. Nutritional supplements were used by $59 \%$ of respondents, with $77 \%$ dose adherence, and $29 \%$ with supplement ingredient knowledge. Half of the respondents $(48 \%)$ planned to carbo-load two-three days before the event, while only five percent used professional advice to scientifically calculate their carbo-loading requirements. $\mathrm{CHO}$ were consumed by $81 \%$ during the event. Hydration preferences during the race were sports drinks (59\%) and water $(22 \%)$; and after the race $45 \%$ preferred a sports drink and $40 \%$ water. Ingredients, taste, colour, and temperature were criteria used to choose a sports drink. Only $18 \%$ of respondents knew to use both colour of urine and thirstiness to determine post-race fluid requirements.

Conclusion: The authors concluded that amateur cyclists had insufficient knowledge regarding nutritional supplement ingredients and usage, $\mathrm{CHO}$ requirements and carbo-loading practices, and hydration strategies before, during and after the event.

Keywords: ergogenic aids, nutritional supplements, carbohydrate use

\section{S Afr J Sports Med 2018;30:1-6. DOI: 10.17159/2078-516X/2018/v30i1a2963}

Nutritional guidelines for sports endurance events suggests the following factors are important in the days before, and the day of the race: optimising muscle glycogen content and hydration status pre-event, carbohydrate feeding and fluid replacement during the event, and the role of supplements (sports foods/drinks and ergogenic aids) for enhancing competition performance. ${ }^{[1]}$ Therefore the authors sought to understand how amateur cyclists (i.e. any cyclist not receiving monetary or apparel support from an outside source), understood and interpreted these guidelines.

Nutritional supplements receive much attention ${ }^{[2,3]}$ with manufacturers pitching the market to accentuate their additional value to health and performance. ${ }^{[4,5]}$ The use of dietary supplements is widespread among athletes. ${ }^{[2]}$ Many amateur and professional athletes may be influenced by the overwhelming industry presence and advertising appeal regarding sports supplementation. In an attempt to enhance and gain an additional performance edge beyond the effects obtained through training, these athletes may consider the use of dietary supplements or engineered foods. ${ }^{[2,3]}$ Athletes may also receive advice from many well-meaning supporters and friends. The scientific evidence in support of these claims is often vague, and it makes the use of the substance controversial and confusing. This leaves the athletes, and especially the amateur athletes, vulnerable to misinformation and risk in terms of the safety, validity, and efficacy of dietary supplements. ${ }^{[3]}$

Carbohydrate $(\mathrm{CHO})$ usage before, during and after endurance events has received ample attention and scrutiny in the past. ${ }^{[1,4]}$ It has been confirmed that $\mathrm{CHO}$ intake plays an intricate role in optimising glycogen storage in muscle before exercising, during, and in the rapid replenishment of muscle glycogen after exercise. ${ }^{[1,4]}$ Various guidelines ${ }^{[5,6]}$ are suggested in scientific and non-scientific literature for carbohydrate use before, during and after an event. The question remains whether amateur cyclists adhere to nutritional guidelines, or if they are oblivious to the fact that nutritional dose adherence may enhance their performance and delay fatigue? Recent reports comparing masters and younger triathletes' postexercise dietary practices $[7,8]$, suggest that both have poor knowledge of recommendations for post-exercise carbohydrate intakes, and that the low intakes of carbohydrate and protein by masters athletes may impair acute recovery. ${ }^{[7,8]}$ This may also hold true when comparing males vs. females. ${ }^{[7,8}$

The role hydration strategies play before, during and after exercise is also supported by the literature. ${ }^{[9,10]}$ Recommended general hydration guidelines include starting to exercise hydrated and drinking sufficiently during exercise (to prevent $>2-3 \%$ loss of initial body weight) to avoid dehydration. Drinking strategies should be adjusted individually to sweat loss and sweat electrolyte content ${ }^{[9,10]}$ and exercise duration. It is recommended that endurance athletes strive to start all exercises well hydrated, that athletes should drink according to thirst and maintain a pale urine colour. ${ }^{[9,10]}$

The Momentum 94.7 Cycle Challenge in South Africa is a 94.7 $\mathrm{km}$ fast circular road race, and the world's second-largest individually timed cycle event after the Cape Town Cycle Tour with 27000 to 35000 cyclists entering annually. The age, sex, 
race experience and health status of participants is varied.

The aim of this study was to determine amateur cyclists': 1) nutritional sports supplement knowledge and use, 2) carboloading and usage, and 3) hydration practices before, during and after an endurance event. Males and females were also compared regarding their supplement knowledge and use, and carbo-loading practices. It was hypothesised that there is evidence of ignorance in amateur cyclists regarding sports supplement use, carbo-loading and hydration strategies, during an endurance event participation.

\section{Methods}

\section{Participants}

All 30640 cyclists who entered the $94.7 \mathrm{~km}$ road race during the 2013 Momentum Cycle Challenge in Johannesburg, South Africa, were invited to partake in this cross-sectional study prior to the event. The Momentum 94.7 Cycle Challenge is an annual one-day fast circular route, $1794 \mathrm{~m}$ above sea level, an elevation range between $1316 \mathrm{~m}$ and $1790 \mathrm{~m}$, and a gain of 741 $\mathrm{m}$. The race has one Category $2 \mathrm{climb}$, one Category $3 \mathrm{climb}$, and two Category 5 climbs. This one-day cycle challenge takes place during November, which ensures hot and humid racing conditions. Participants are widespread in terms of age, sex, race experience and health status. Starting time is staggered in blocks of \pm 300 cyclists per block. Cyclists are allocated to different starting times depending on their seeding.

\section{Questionnaire}

The questionnaire was developed by a panel of subject experts to assess the planned supplement, nutritional, and hydration strategies during the 2013 Momentum 94.7 Cycle Challenge. The event organisers were approached via email on using the participants for research purposes and permission was obtained to invite volunteers who wanted to be part of the research. The questionnaire was posted online on the Momentum 94.7 Cycle Challenge website a few days prior to the event. The objective of the questionnaire was explained on the website. All cyclists could complete the survey anonymously and had the option to quit the survey at any stage. Only complete questionnaires $(n=2550)$ were included in the analysis. Professional cyclists were excluded.

A survey with questions and answer options to determine the amateur cyclist's knowledge regarding nutritional supplement use, carbo-loading and hydration strategies, before, during and after an endurance race was introduced. The questionnaire was comprised of four parts:

- Demographic data in terms of age and sex were collected,

- Nutritional supplement (any supplement) usage and knowledge were collected (reasons for use, dose adherence, and knowledge of ingredients) via a dropdown list with an open-ended option,

- Carbo-loading and $\mathrm{CHO}-$ usage were examined (reasons for use, reason for not carbo-loading, type of $\mathrm{CHO}$ used before, during and after the race, and if $\mathrm{CHO}$ requirements were calculated or guessed),

- Hydration knowledge and habits were appraised (the frequency of fluid intake, their preferred type of drink during the race, the type of drink and fluid needs after the race).

The Ethics Committee at the Faculty of Health Sciences of the University of Pretoria approved the protocol for the study. Consent to participate in the Momentum 94.7 Cycle Challenge, as well as completing the research study questionnaire, was obtained during online race entry by the race organisers. Parents of children aged 12-18 years were requested to give consent to be part of the research study at the time of the online entry for the race.

\section{Data analysis}

Data were analysed using IBM SPSS Statistics version 22. The data set was summarised univariately, as well as bivariately by age and sex, to gain insight into participants' habits and levels of usage and knowledge. Chi-square tests $\left(\chi^{2}\right)$ were performed to assess interactions between males and females with regard to supplement usage and knowledge, as well as carbo-loading. In the case of significant results, the adjusted residual is reported and the expected frequencies under the null hypothesis of no interaction compared to the observed counts to facilitate interpretation of the interactions.

\section{Results}

\section{Demographics}

The demographics of the cyclists' age and sex are reported in Table 1 . The response rate was $8 \%$ ( $75 \%$ male and $25 \%$ female), which represented the overall race demographics of $77 \%$ male and $23 \%$ female entrants. The largest response to the nutritional questionnaire came from the 25-45 year age group.

\section{Supplement usage}

Nutritional supplement usage was indicated by $61 \%$ of the respondents. As seen in Figure 1, the main reason for supplement usage was to aid in recovery $(45 \%)$, followed by being recommended by either a friend $(35 \%)$, or a dietician $(18 \%)$. Another popular reason for supplement usage was the cyclists' concern regarding insufficient training (15\%).

Table 1. Demographics of race entrants vs. questionnaire respondents divided by age groups and sex

\begin{tabular}{lcc}
\hline & $\begin{array}{c}\text { All Race Entrants } \\
(\mathbf{n}=30640)\end{array}$ & $\begin{array}{c}\text { Questionnaire } \\
\text { Respondents } \\
(\mathbf{n}=2550)\end{array}$ \\
\hline Age Groups & $\mathbf{n} \mathbf{( \% )}$ & $\mathbf{n} \mathbf{( \% )}$ \\
12-18 years & $1057(3)$ & $5(0.2)$ \\
19-24 years & $2020(7)$ & $117(5)$ \\
$25-30$ years & $4835(16)$ & $368(14)$ \\
$31-35$ years & $4656(15)$ & $378(15)$ \\
$36-40$ years & $5019(16)$ & $380(15)$ \\
$41-45$ years & $4742(15)$ & $414(16)$ \\
$46-50$ years & $3438(11)$ & $327(13)$ \\
$51-55$ years & $2434(8)$ & $251(10)$ \\
56 years + & $2439(8)$ & $310(12)$ \\
Sex & $\mathbf{n} \mathbf{( \% )}$ & $\mathbf{n} \mathbf{( \% )}$ \\
Female & $7192(23)$ & $641(25)$ \\
Male & $23448(77)$ & $1909(75)$ \\
\hline$n=$ total number of participants, \% $=$ Proportional frequency of participants \\
in each category \\
$\begin{array}{l}\text { Age group categories: Indicated as received from the Momentum 94.7 Cycle } \\
\text { Challenge race organisers. }\end{array}$
\end{tabular}


Advertising did not seem to play a prominent role in their decision-making. Dose adherence as stated on the packaging label or pamphlet was followed by $75 \%$ of athletes. Only $2.5 \%$ admitted to taking more than the recommended dosage specified. There was a significant interaction between males and females with regard to nutritional supplement usage $\left(\chi^{2}(1)=\right.$ 4.681, $\mathrm{p}=0.031$; Adjusted residual $=-2.2$ ). Interestingly, fewer females used nutritional supplements than was expected under the null hypothesis of no interaction. There was no significant interaction between males and females regarding their knowledge of supplement ingredients $\left(\chi^{2}(1)=0.102, p=\right.$ $0.749)$. Almost half (47\%) of respondents were familiar with the ingredients in their sports supplement. Ingredients frequently selected by this sample of athletes were $\mathrm{CHO}$, caffeine, whey protein, amino acids, magnesium and Vitamin C.

\section{CHO-intake strategies}

Approximately two-three days prior to the race, $48 \%$ of respondents deliberately intended to carbo-load. There was no significant interaction between males and females with regard to carbo-loading habits $\left(\chi^{2}(1)=0.437 ; \mathrm{p}=\right.$ $0.509)$. More than half of the participants in the 25-30 year age group used carboloading. In all other age groups less than half of the participants used carboloading. However, there was no significant difference $(\mathrm{p}=$ 0.709 ) between any of the age groups (Figure 2).

Of the $52 \%$ respondents who did not carbo-load prior to the event, they claimed that the main reason therefore

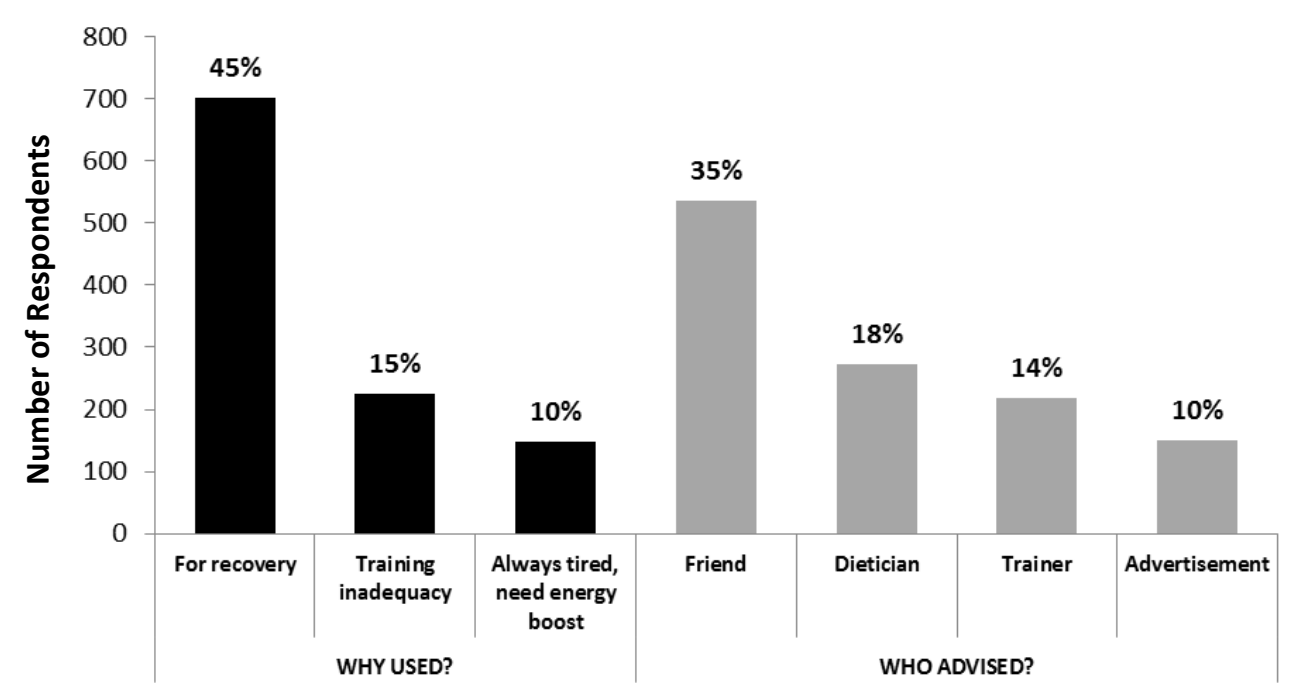

Fig. 1. Reasons for supplement use, and source of information. (Respondents could select multiple reasons for using supplements.)

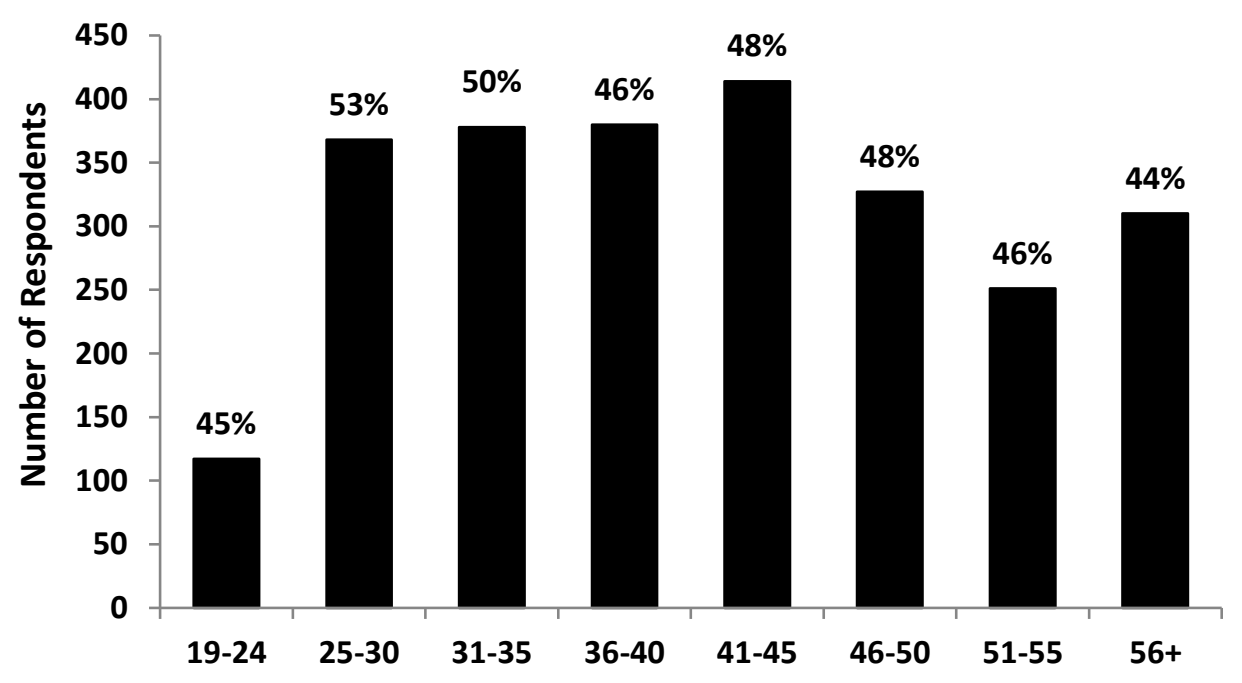

Fig. 2. Proportion of participants in each age group who indicated to $\mathrm{CHO}$-loading prior to the event $(n=2545) .(<18$ years omitted due to small sample size. $)$

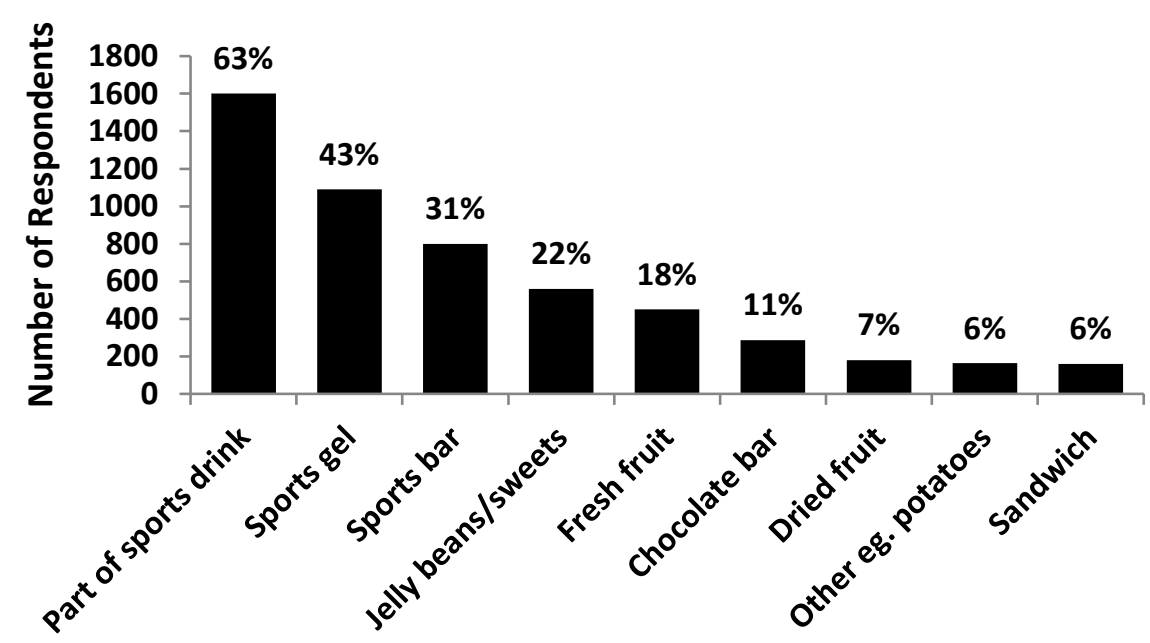

Fig. 3. Preferred source for CHO-replacement during the race. (Respondents could select multiple CHO- replacement sources.) 
was that they did not need it (69\%), followed by the fear of weight gain $(16 \%)$, and lastly, experiencing gastrointestinal tract (GIT) side effects $(12 \%)$. During the race, $81 \%$ of the cyclists planned to use a $\mathrm{CHO}$ preferably as a sports drink, sports gel or sports bar (Figure 3), while whole food choices, for example, sweets, fruit, sandwiches and potatoes were less preferred.

After the race, cyclists preferred the $\mathrm{CHO}$ to be a sports drink, or part of a meal. Most of the cyclists who carbo-loaded $(35 \%)$ used the formula given on their product packaging (not taking body weight into account). Athletes took their carbo-loading advice from a friend $(28 \%)$, and others obtained their advice from sports magazines $(11 \%)$ and the internet $(20 \%)$,

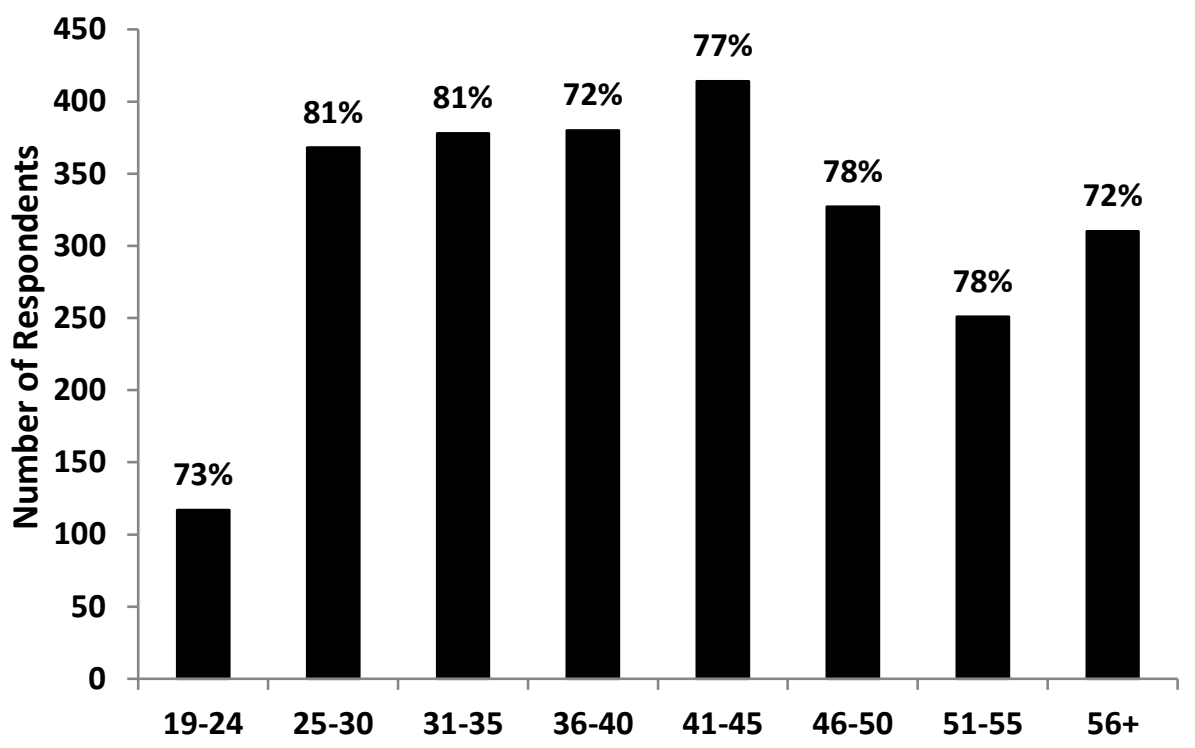
respectively. Only $5 \%$ of the cyclists reported that they made use of professional advice and calculated their carbo-loading according to a nutritional scientific formula based on their body weight (8-12g CHO/kg body weight/day). There was no statistical significant difference $(p=0.116)$ between age groups regarding methods used to calculate $\mathrm{CHO}$ requirements (Figure 4). There was no significant interaction between males and females with regard to carbo-loading requirement knowledge $\left(\chi^{2}(2)=0.2861, p=0.8667\right)$.

\section{Hydration strategies}

The frequency of fluid intake during the race was as follows: $38 \%$ of respondents planned to drink fluids every 15 minutes and $28 \%$ every 30 minutes. The preferred fluids during the race were a sports drink $(59 \%)$, and water $(22 \%)$. The criteria used to choose a sports drink were based on ingredients $(29 \%)$, taste $(28 \%)$, and on a friend's recommendations $(19 \%)$. With regard to ingredient knowledge, the most sought-after ingredients preferred by the cyclists in their sports drink, were electrolytes $(33 \%), \mathrm{CHO}(27 \%)$, vitamins and minerals $(16 \%)$, and proteins $(12 \%)$. Half of the respondents $(55 \%)$ did not know the percentage of $\mathrm{CHO}$ in their preferred sports drink, and $43 \%$ did not know the type of $\mathrm{CHO}$. After the race, $45 \%$ preferred a sports drink and $40 \%$ water as their choice of fluid. Only $18 \%$ of respondents were aware of using both the colour of their urine and their feeling of thirst to determine their post-race fluid requirements.

\section{Discussion}

This study investigated the intended use of supplements, $\mathrm{CHO}$ and hydration strategies by amateur cyclists participating in a $94.7 \mathrm{~km}$ one day endurance cycle event. The participants that responded to the online nutritional questionnaire were representative with regard to age and sex. The main findings of this research showed that the majority of respondents used supplements (59\%) and adhered to the

dose $(77 \%)$, although only $29 \%$ were familiar with the specific ingredients in their preferred sports supplement. Half of the respondents carbo-loaded, but a mere 5\% used professional advice to scientifically calculate their requirements. The main reasons given for not carbo-loading were ignorance, fear of gaining weight and GIT side effects. Hydration preferences during and after the race were a sports drink and water respectively. Only a few respondents knew to use the colour of their urine and the feeling of thirst to determine post-race fluid requirements.

Research on the supplement use strategies of professional athletes ${ }^{[2]}$ is more often reported than in recreational athletes. ${ }^{[11]}$ Most studies confirmed that athletes, regardless of sex and age, do use supplements. ${ }^{[1,2,3]}$ The main reasons for professional athletes taking supplements were enhanced recovery and greater muscle strength, ${ }^{[12]}$ whereas a study by Morrison compiled on recreational supplement users indicated the reasons for taking supplements varied considerably. ${ }^{[13]}$ The use of supplements and the reasons given for their usage corresponds to the findings in this study on recreational cyclists. This study also found that most of the athletes adhered to the dose as indicated on the package insert, while a few admitted to increasing the recommended dose specified.

Contrary to this study's findings, a previous study on commercial gym users indicated that the majority of them get information regarding the use of supplements from magazines $(66 \%)$, family or friends $(63 \%)$, health food store staff $(56 \%)$, books $(55 \%)$, the media $(46 \%)$, and personal trainers $(39 \%)$. Only a small number of the abovementioned athletes visited a physician $(34 \%)$, dietitian $(28 \%)$ or pharmacist $(17 \%)$ for advice on the supplements they use, which is in agreement with the findings of this research. ${ }^{[13]}$ With information being readily available, consumers are expected to be more aware, educated and health conscious about the supplements they use. However, this present study revealed that only $29 \%$ of respondents have an adequate knowledge of supplement 
ingredients, confirming the need for more appropriate education in this regard.

The authors found a significant interaction between males and females, with fewer females reporting the use of nutritional supplementation during the $94.7 \mathrm{~km}$ Momentum Cycle Challenge. This is in accordance with a study conducted in Canada on university students that found only $51 \%$ male athletes and $43 \%$ female athletes used a protein supplement, while in the non-athletic group only $23 \%$ males and $17 \%$ females used protein supplements. ${ }^{[12]}$

Numerous studies have been done on carbo-loading techniques to validate the ergogenic possibilities of various methods and the duration of loading. ${ }^{[1,4-6]}$ Research indicates that many athletes who attempt to carbo-load fail to achieve their goal. ${ }^{[7,8]}$ Therefore many opt not to carbo-load, as proven by the majority of respondents in this study that did not plan to carbo-load before this event. Carbo-loading also causes a body mass increase of approximately two $\mathrm{kg}$, and additionally, it is important to have high-CHO, low-fibre, and low-fat foods. ${ }^{[14]}$ This present study revealed that $16 \%$ of respondents opted not to carbo-load for the fear of weight gain, and $12 \%$ due to experiencing GIT side effects. This again highlights the need for nutritional education in this regard.

During exercise, low levels of muscle glycogen or blood glucose may be contributing factors in the premature onset of fatigue. ${ }^{[4,6]}$ Consuming $\mathrm{CHO}$ before and during prolonged exercise may help to delay exhaustion during endurance events. ${ }^{4-6]}$ Generally, the regular muscle glycogen stores of elite and amateur athletes are sufficient to sustain exercise duration of up to $60-90$ minutes. However, in longer lasting events of intermittent high-intensity exercise lasting more than 90 minutes, athletes may experience fatigue due to exhaustion of muscle glycogen stores. ${ }^{[4-6]}$ Birkenhead and Slater ${ }^{[15]}$ noted that the food choices athletes make are motivated by taste, convenience, nutrition knowledge and beliefs, and are also influenced by physiological, psychological, social and economic factors. The $81 \%$ respondents in this present study that planned to use a $\mathrm{CHO}$ during the race, preferred cheap, convenient, and readily available sources.

A study by Hottenrott et al.[16] investigated athletes' selfchosen dietary strategies compared to a scientifically formulated strategy, in relation to their performance. They concluded that performance was better in the group that used scientific dietary strategies. Although more than a third of the cyclists carbo-loaded according to the formula given on their product packaging, few of the respondents in this study calculated their carbo-loading requirements according to the formula of a dietitian or doctor. This correlates with results from a study done on non-elite multisport endurance athletes, which concluded that many of the athletes do not meet the current recommendations for carbo-loading during an event, emphasising the need for better targeted nutritional education. ${ }^{[7,8]}$

Less than a third (27\%) of the Momentum 94.7 Cycle Challenge cyclists examined the $\mathrm{CHO}$ content of their sports drinks. Although current research ${ }^{[4-6]}$ suggests that $6-10 \%$ $\mathrm{CHO}$ solutions and hydration solutions containing multiple- transportable $\mathrm{CHO}$ may be effective for athletes who need $\mathrm{CHO}$ replacement during exercise, only half of the respondents in this study knew the percentage of the $\mathrm{CHO}$ in their preferred sports drink, and less than half of the respondents knew the type of $\mathrm{CHO}$ in their chosen sports drink. Contrary to expectations by the athlete, their lack of knowledge may lead to ineffective carbohydrate replacement. ${ }^{[7,8]}$

Electrolytes were the most sought-after ingredient (33\%) in selecting a sports beverage in this study. It has been proven that electrolyte replacement is generally not needed during shortterm exercise. It may, however, be beneficial during very lengthy exercise periods, or if the athlete has a high sweat rate. ${ }^{[9,10]}$ In this study, proteins were one of the ingredients the cyclists regarded essential in their sports drink.

The past few decades have seen an evolution in fluid intake guidelines for endurance and ultra-endurance events. ${ }^{[9,10]}$ The recommended strategies have changed from encouraging to drink as much as possible, to drinking only when thirsty. The frequency of fluid intake in this study, as indicated by the cyclists, was quite high. Unfortunately, volume was not considered, thus limiting the discussion on hydration.

Further limitations to this study include the questionnaire being non-compulsory to all participants in the Momentum 94.7 Cycle Challenge, and although the response rate represented the overall event demographics, it was only completed by individuals prepared to make the effort. Recall bias based on memory recollection of the participants may also have played a role in the response to questions. Furthermore, an interesting future focus will be to include the performance level of the cyclists to determine whether those participating at a higher level have a different level of knowledge, which is a current shortcoming in this study.

\section{Conclusion}

It seems that amateur cyclists participating in the 2013 Momentum 94.7 Cycle Challenge have inadequate knowledge regarding the supplements they prefer using, as well as wideranging carbo-loading and $\mathrm{CHO}$-usage habits. They also have insufficient knowledge regarding optimal hydration strategies. There is thus a real need for education regarding supplement usage and knowledge, $\mathrm{CHO}$ ingestion habits and hydration practices, in amateur cyclists.

Acknowledgements: The questionnaire was funded by Momentum, one of the sponsors of the Momentum 94.7 Cycle Challenge.

Authorships: Study design: Dr I M Sparks and Prof D C Janse van Rensburg. Data collection: Via electronic questionnaire. Data analysis: Dr L Fletcher. Data interpretation and manuscript preparation: Dr IM Sparks, Prof DC Janse van Rensburg, Dr L Fletcher and A Jansen van Rensburg.

Declarations: All authors approved the final version of the paper, and have no conflict of interest to declare.

Statement: The protocol was approved by the Ethics Committee of the University of Pretoria, South Africa. 


\section{References}

1. Thomas DT, Erdman KA, Burke LM. American College of Sports Medicine Joint Position Statement. Nutrition and Athletic Performance.Med Sci Sports Exerc 2016;48(3):543-568. [doi: 10.1249/MSS.0000000000000852]

2. Knapik JJ, Steelman RA, Hoedebecke SS, et al. Prevalence of dietary supplement use by athletes: Systematic review and meta-analysis. Sports Med 2016;46(1):103-123. [doi: 10.1007/s40279-015-0387-7]

3. Buell JL, Franks R, Ransone J, et al. National Athletic Trainers' Association position statement: evaluation of dietary supplements for performance nutrition. J Athl Train 2013;48(1):124-136. [doi: 10.4085/1062-6050-48.1.16]

4. Beelen M, Cermak NM, van Loon LJ. Performance enhancement by carbohydrate intake during sport: effects of carbohydrates during and after high-intensity exercise. Nederlands Tijdschrift voor Geneeskunde. 2015;159:A7465.

5. Mayo JJ. Carbohydrate intake for peak performance: practical guidelines for the endurance athlete. AMAA Journal 2015;28(3):8-11.

6. Jeukendrup AE. Periodized nutrition for athletes. Sports Med 2017;47(Suppl 1):51-63. [doi: 10.1007/s40279-017-0694-2]

7. Masson G, Lamarche B. Many non-elite multisport endurance athletes do not meet sports nutrition recommendations for carbohydrates. Appl Physiol Nutr Metab 2016;41(7):728-734. [doi: 10.1139/apnm-2015-0599]

8. Heikura IA, Stellingwerff T, Mero AA, et al. A mismatch between athlete practice and current sports nutrition guidelines among elite female and male middle-and long-distance athletes. Int J Sport Nutr Exerc Metab 2017;27(4):351-360. [doi: 10.1123/ijsnem.2016-0316]
9. Armstrong LE, Johnson EC, McKenzie AL, et al. Ultraendurance cycling in a hot environment: thirst, fluid consumption, and water balance. J Strength Cond Res 2015;29(4):869-876. [doi: 10.1519/JSC.0000000000000822]

10. American College of Sports Medicine, Sawka MN, Burke LM, et al. American College of Sports Medicine position stand. Exercise and fluid replacement. Med Sci Sports Exerc 2007;39(2):377-390. [doi: 10.1249/mss.0b013e31802ca597]

11. Tsitsimpikou C, Chrisostomou N, Papalexis P,et al. The use of nutritional supplements among recreational athletes in Athens, Greece. Int J Sport Nutr Exerc Metab 2011;21(5):377-384.

12. Kristiansen M, Levy-Milne R, Barr S, et al. Dietary supplement use by varsity athletes at a Canadian university. Int J Sport Nutr Exerc Metab 2005;15(2):195-210.

13. Morrison LJ, Gizis F, Shorter B. Prevalent use of dietary supplements among people who exercise at a commercial gym. Int J Sport Nutr Exerc Metab 2004;14(4):481-492.

14. Potgieter S. Sport nutrition: A review of the latest guidelines for exercise and sport nutrition from the American College of Sport Nutrition, the International Olympic Committee and the International Society for Sports Nutrition. S Afr J Clin Nutr 2013;26(1):6-16.

15. Birkenhead KL, Slater G. A review of factors influencing athletes' food choices. Sports Med 2015;45(11):1511-1522. [doi: 10.1007/s40279-015-0372-1]

16. Hottenrott $K$, Hass E, Kraus M, et al. A scientific nutrition strategy improves time trial performance by $\approx 6 \%$ when compared with a self-chosen nutrition strategy in trained cyclists: a randomized cross-over study. Appl Physiol Nutr Metabol 2012;37(4):637-645. [doi: 10.1139/h2012-028] 